\title{
Protection against hypoxia-induced blood-brain barrier disruption: changes in
} intracellular calcium

\author{
Rachel C. Brown, Karen S. Mark, Richard D. Egleton, and Thomas P. Davis \\ Department of Pharmacology, University of Arizona, Tucson, Arizona 85724
}

Submitted 26 August 2003; accepted in final form 15 December 2003

Brown, Rachel C., Karen S. Mark, Richard D. Egleton, and Thomas P. Davis. Protection against hypoxia-induced blood-brain barrier disruption: changes in intracellular calcium. Am J Physiol Cell Physiol 286: C1045-C1052, 2004. First published December 18, 2003; 10.1152/ajpcell.00360.2003.-Tissue damage after stroke is partly due to disruption of the blood-brain barrier (BBB). Little is known about the role of calcium in modulating BBB disruption. We investigated the effect of hypoxic and aglycemic stress on BBB function and intracellular calcium levels. Bovine brain microvessel endothelial cells were treated with A-23187 to increase intracellular calcium without hypoxia or treated with a calcium chelator (BAPTA) or calcium channel blockers (nifedipine or SKF-96365) and $6 \mathrm{~h}$ of hypoxia. A-23187 alone did not increase paracellular permeability. Hypoxia increased intracellular calcium, and hypoxia or hypoxiaaglycemia increased paracellular permeability. Treatment with nifedipine and SKF-96365 increased intracellular calcium under normoglycemic conditions, instead of blocking calcium influx, and was protective against hypoxia-induced BBB disruption under normoglycemia. Protection by nifedipine and SKF-96365 was not due to antioxidant properties of these compounds. These data indicate that increased intracellular calcium alone is not enough to disrupt the BBB. However, increased intracellular calcium after drug treatment and hypoxia suggests a potential mechanism for these drugs in BBB protection; nifedipine and SKF-96365 plus hypoxic stress may trigger calcium-mediated signaling cascades, altering BBB integrity.

nifedipine; SKF-96365; ischemia; permeability; fura 2

IONIC HOMEOSTASIS of the central nervous system (CNS) is critical for normal brain function and is dependent on the blood-brain barrier (BBB). This barrier, which isolates the brain from the peripheral circulation, is located at the level of the cerebral microvessel endothelial cells. The BBB is characterized by tight cell-cell contacts with limited paracellular diffusion and reduced fluid-phase endocytosis (56). The presence of specific endothelial cell transporters for ions, peptides, and nutrients allows for strict regulation of the CNS microenvironment. Disruption of the BBB occurs in a number of pathological conditions, including Alzheimer's disease, diabetes, multiple sclerosis, inflammatory pain, and stroke $(1,4,28$, $33,39)$, and can contribute to CNS edema formation $(45,52)$.

The restrictive nature of the BBB is due to tight junctions (TJ) formed between adjacent endothelial cells in the brain capillaries (42). The TJ restricts ion flux and paracellular diffusion of macromolecules. A number of the proteins that compose $\mathrm{TJ}$ have been identified and extensively characterized, including the claudins $(26,54)$, occludin (25), and zonula occludens (ZO)-1, -2 , and -3 , which interact with claudins and

Address for reprint requests and other correspondence: T. P. Davis, Dept. of Pharmacology, PO Box 24-5050, The Univ. of Arizona College of Medicine, 1501 N. Campbell Ave., Tucson, AZ 85724-5050 (E-mail: davistp@u.arizona.edu). occludin $(37,53)$. Stabilization of TJ involves a complex network of the transmembrane proteins occludin and claudins, linked to the actin cytoskeleton by accessory $\mathrm{ZO}$ proteins, which mediate this linkage by binding the cytoplasmic tails of occludin and claudin to actin (32).

Calcium is critical to normal BBB function (13). Endothelial cells incubated in calcium-free medium had decreased transendothelial electrical resistance readings and increased monolayer paracellular permeability (58), indicating disruption of TJ. In brain microvessel endothelial cells in culture, hypoxia caused an increase in intracellular calcium (35, 41, 55), whereas blockade of calcium flux (1) or blockade of calciumregulated signaling cascades (55) prevented hypoxia-induced disruption of BBB monolayer integrity.

Stroke is the third leading cause of death in the United States and is the leading cause of long-term disability, affecting $\sim 500,000$ patients every year (50). Although the neuronal damage associated with stroke is due in part to lack of oxygen and nutrients during an ischemic event, reperfusion, the restoration of oxygen supply, and subsequent production of free radicals are also major contributors in mediating neuronal damage and death (50). Ischemic damage to blood vessels, in particular to the brain microvessel endothelial cells that make up the BBB, results in deregulation of ion flux and increased ion permeability into the brain, leading to a net influx of solutes and water and subsequent edema formation. Several studies found increased paracellular permeability after hypoxic or ischemic insult $(1,5,30)$, suggesting disruption of the TJ. Transient ischemia in rats increased extravasation of Evans blue albumin, a normally impermeable marker (5). This increase in paracellular permeability is correlated with the degree of tissue damage. A number of in vitro models have been used to investigate the effects of hypoxia and aglycemia on the BBB. Hypoxia and aglycemia increased the paracellular permeability of bovine brain microvessel endothelial cell (BBMEC) monolayers (1). Nifedipine (NIF), an L-type calcium channel antagonist, and SKF-96365 (SKF), an inhibitor of some store-operated calcium channels, blocked this increase in permeability, indicating a potential role for calcium in mediating some of the hypoxia- and/or aglycemia-induced alterations in BBB integrity. In the present study, we investigated the effect of the calcium channel blockers NIF and SKF on intracellular calcium levels, BBMEC monolayer permeability, and reactive oxygen species (ROS) generation to elucidate potential mechanisms by which calcium might be involved in mediating BBB integrity and function after a hypoxic and/or aglycemic insult.

The costs of publication of this article were defrayed in part by the payment of page charges. The article must therefore be hereby marked "advertisement" in accordance with 18 U.S.C. Section 1734 solely to indicate this fact. 


\section{MATERIALS AND METHODS}

In vitro $B B B$ model. $\mathrm{BBMEC}$ were isolated from the gray matter of the cerebral cortex of fresh bovine brains obtained from the University of Arizona Meat Laboratory and cryopreserved as previously described $(3,49)$. Isolated cells were seeded onto collagen-fibronectincoated Transwell filters or slides and grown in MEM-Ham's F-12 with $10 \%$ equine serum, $50 \mu \mathrm{g} / \mathrm{ml}$ gentamicin, $2.5 \mu \mathrm{g} / \mathrm{ml}$ amphotericin B, and $100 \mu \mathrm{g} / \mathrm{ml}$ heparin. All BBMEC used for these studies were primary cultured cells from passage 0 , which have been shown to maintain excellent $\mathrm{BBB}$ characteristics in vitro $(2,66)$. Cultures in Transwell filters were incubated with $\mathrm{C} 6$ astrocyte-conditioned medium in the basolateral chamber for 3 days before the start of experiments (14).

Calcium ionophore treatment and hypoxic stress. BBMEC monolayers were treated with $5 \mu \mathrm{M}$ A-23187, a calcium ionophore, for $6 \mathrm{~h}$ to increase levels of intracellular calcium. Some monolayers were pretreated before the addition of A-23187 for 30 min with $10 \mu \mathrm{M}$ BAPTA-AM (BAPTA), a cell-permeable calcium chelator, to inhibit the increase in intracellular calcium due to the calcium ionophore. Monolayer permeability was assayed as described below.

For hypoxic experiments, BBMEC monolayers were subjected to $6 \mathrm{~h}$ of hypoxia under different treatment conditions. Monolayers were incubated in normoglycemic RPMI 1640 (Life Technologies, Rockville, MD) and treated with $10 \mu \mathrm{M}$ BAPTA, $100 \mathrm{nM}$ NIF, or $100 \mathrm{nM}$ SKF; drugs were added $30 \mathrm{~min}$ before the onset of hypoxic stress. Other monolayers were subjected to hypoxic stress + aglycemia; these cells were incubated in modified RPMI 1640 without glucose (Life Technologies, Rockville, MD) and were treated with BAPTA, NIF, or SKF. Hypoxic stress was achieved by incubating the monolayers in a humidified, gas-controlled hypoxic workstation (Coy Laboratory Products, Grass Lake, $\mathrm{MI})$ at $37^{\circ} \mathrm{C}$ with $99 \% \mathrm{~N}_{2}-1 \% \mathrm{O}_{2}$. At the end of the hypoxic period, the medium was removed and $\mathrm{PO}_{2}$ levels were measured with a blood gas analyzer (ABL 505, Radiometer, Copenhagen, Denmark) to confirm hypoxia. $\mathrm{PO}_{2}$ in medium from normoxic samples was $150.2 \pm 4.5 \mathrm{mmHg} ; \mathrm{Po}_{2}$ in hypoxic medium was $44.1 \pm 1.6 \mathrm{mmHg}$. Monolayers were assayed for changes in intracellular calcium levels or functional permeability (see below).

Measurement of intracellular calcium. To measure intracellular calcium, BBMEC were plated into 96-well plates. When confluent, cells were incubated in $5 \mu \mathrm{M}$ fura 2-AM alone or with BAPTA, NIF, or SKF for $30 \mathrm{~min}$ at $37^{\circ} \mathrm{C}$. After the incubation period, fura 2-AM was removed, and medium containing appropriate drugs was added. Readings were taken at various times over a 6-h period of incubation as described below.

For changes in intracellular calcium levels over a hypoxic stress period, an initial reading was obtained, and plates were transferred to the hypoxic chamber for treatment. The microplate was covered with nonpermeable adhesive membrane during fluorescence readings to maintain hypoxic conditions. At each time point, the plate was covered with a nonpermeable adhesive membrane and removed from the hypoxic chamber for fluorescence readings. At the end of the reading, the plate was returned to the chamber and the adhesive membrane was removed; there was no reoxygenation of the medium or cells during the reading. Changes in intracellular calcium levels were determined by measuring fura 2 fluorescence at $340 \mathrm{~nm}$ excitation and $510 \mathrm{~nm}$ emission on a GENios microplate reader (Tecan, Research Triangle Park, NC). Calcium-insensitive fura 2 fluorescence was measured at $380 \mathrm{~nm}$ excitation and $510 \mathrm{~nm}$ emission, and results were expressed as percentage of time 0 control ratio of fluorescence at $340 \mathrm{~nm}$ to fluorescence at $380 \mathrm{~nm}$. Measurements were obtained as described above at various times over the 6-h hypoxic stress period. In a related experiment, cells were incubated with NIF and SKF, and basal fura 2-AM fluorescence was measured before the addition of $100 \mathrm{nM}$ bradykinin. Changes in intracellular calcium fluorescence were measured as described above. After the time course of fura 2 fluorescence was plotted for each treatment, area under the curve (AUC) was determined, and values were statistically analyzed.

Permeability measurements. Permeability studies used $\left[{ }^{14} \mathrm{C}\right] \mathrm{su}-$ crose, a normally low-permeability marker, to determine paracellular flux across BBMEC monolayers. Apical-to-basolateral flux was determined by dividing picomoles of radioactive marker appearing in the receiver chamber by the time in minutes $(1,49)$. The apparent permeability coefficient (PC) was calculated as follows

$$
\mathrm{PC}=\operatorname{flux} /\left(A * \mathrm{C}_{\text {Do }}\right)
$$

where flux is the slope of the line, $A$ is the area of the membrane, and $\mathrm{C}_{\mathrm{Do}}$ is the initial donor concentration of radioactive marker.

Measurement of ROS. BBMEC were plated in 96-well plates and incubated with normoglycemic medium, aglycemic medium, or aglycemic medium $+\operatorname{drug}(100 \mathrm{nM}$ NIF or $100 \mathrm{nM} \mathrm{SKF})$. At $1 \mathrm{~h}$ before the end of the hypoxic stress, cells were incubated in $50 \mu \mathrm{M} \mathrm{2} 2^{\prime}, 7^{\prime}$ dichlorofluorescein diacetate (Calbiochem, San Diego, CA), a fluorescent probe for measuring oxidative stress (12). Production of ROS was measured by obtaining fluorescence readings at $485 \mathrm{~nm}$ of excitation and $535 \mathrm{~nm}$ of emission on the GENios microplate reader after $6 \mathrm{~h}$ of normoxia, hypoxia, or hypoxia followed by $30 \mathrm{~min}$ of reoxygenation.

Statistics. Values are means \pm SE. Results were analyzed with oneor two-way ANOVA as appropriate and then with multiple pairwise comparisons with Tukey's test using Sigma Stat 2.03 (SPSS, Chicago, IL). Significance was defined as $P<0.05$.

\section{RESULTS}

We used BBMEC monolayers as a model system to investigate the effects of increasing intracellular calcium levels or of hypoxic and/or aglycemic stress on BBB functional integrity. BBMEC were exposed to various treatment conditions before determination of intracellular calcium levels and monolayer paracellular permeability.

A-23187, a calcium ionophore, did not alter monolayer permeability. Incubation of $\mathrm{BBMEC}$ monolayers in $5 \mu \mathrm{M}$ A-23187 or $10 \mu \mathrm{M}$ BAPTA did not change $\left[{ }^{14} \mathrm{C}\right]$ sucrose permeability compared with untreated control (Fig. 1). However, treatment with A-23187 + BAPTA significantly in-

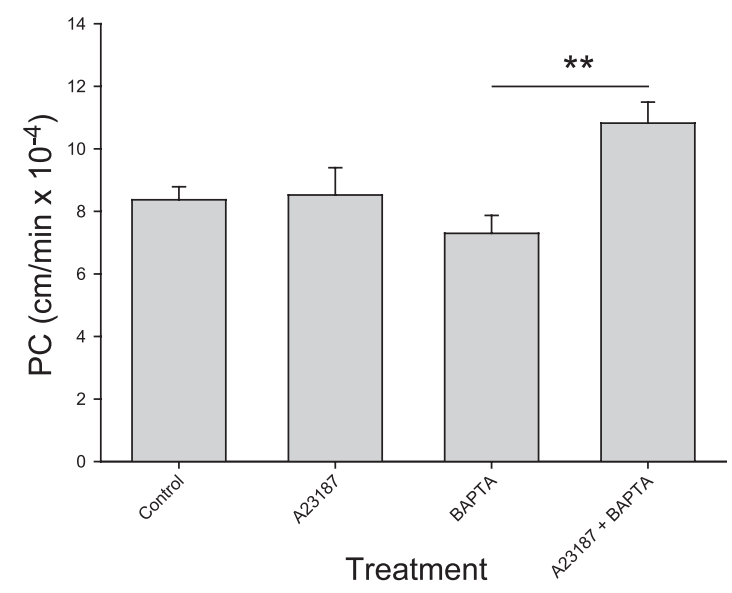

Fig. 1. A-23187 has no effect on bovine brain microvessel endothelial cell (BBMEC) monolayer permeability. BBMEC monolayers were incubated with $5 \mu \mathrm{M}$ A-23187 and/or $10 \mu \mathrm{M}$ BAPTA, and $\left[{ }^{14} \mathrm{C}\right]$ sucrose permeability was assayed. Neither A-23187 nor BAPTA treatment affected BBMEC permeability. When monolayers were incubated with A-23187 + BAPTA, permeability was significantly increased compared with BAPTA treatment alone. PC, permeability coefficient. Values are means $\pm \mathrm{SE} ; n=5-6$. $* * P=0.01$. 
creased BBMEC monolayer permeability compared with BAPTA alone $\left(F_{3,22}=5.029, P=0.01\right)$.

Hypoxic stress increased intracellular calcium; treatment with NIF or SKF further increased levels of intracellular calcium over the course of hypoxic stress. BBMEC monolayers were exposed to $6 \mathrm{~h}$ of $1 \%$ oxygen in normoglycemic or aglycemic medium, and fura 2 fluorescence was measured as an indicator of changes in intracellular calcium. A characteristic pattern of changes in intracellular calcium in normoglycemic or aglycemic medium was observed (Fig. 2). During the $1 \mathrm{st} \mathrm{h}$ of hypoxia, the stress appeared to induce long-term oscillations in intracellular calcium levels, with several apparent peaks. These initial oscillations appeared to subside, with intracellular calcium levels returning to near baseline (time 0 ) levels by $2 \mathrm{~h}$. There was a subsequent increase in intracellular calcium at $4 \mathrm{~h}$ of hypoxic stress that returned to normal at $5 \mathrm{~h}$ and increased again at $6 \mathrm{~h}$ of hypoxic stress, perhaps as part of longer-term oscillatory behavior.

Using similar assay conditions, we examined the effects of BAPTA, NIF, and SKF, alone and in combination, on intracellular calcium levels. Data were expressed as percentage of time $O$ control samples, and the AUC was calculated and analyzed by two-way ANOVA (Fig. 3). Drug treatment had a significant effect on intracellular calcium levels $\left(F_{6,604}=\right.$ $2.298, P=0.033$ ); furthermore, there was a significant interaction between drug treatment and glycemic condition $\left(F_{6,604}=4.269, P<0.001\right)$. Under hypoxic-normoglycemic conditions, treatment with NIF and/or SKF increased levels of intracellular calcium, contrary to the predicted effects of these drugs as calcium channel blockers (Fig. 3A). Treatment with BAPTA decreased intracellular calcium levels, while treatment with BAPTA + NIF or SKF abolished the increase in intracellular calcium due to NIF or SKF alone. In contrast, under aglycemic conditions (Fig. 3B), treatment with the drugs alone after $6 \mathrm{~h}$ of hypoxic stress had no effect on calcium levels, but treatment with drug combinations (BAPTA + NIF or BAPTA + SKF) led to unexpectedly increased intracellular calcium.

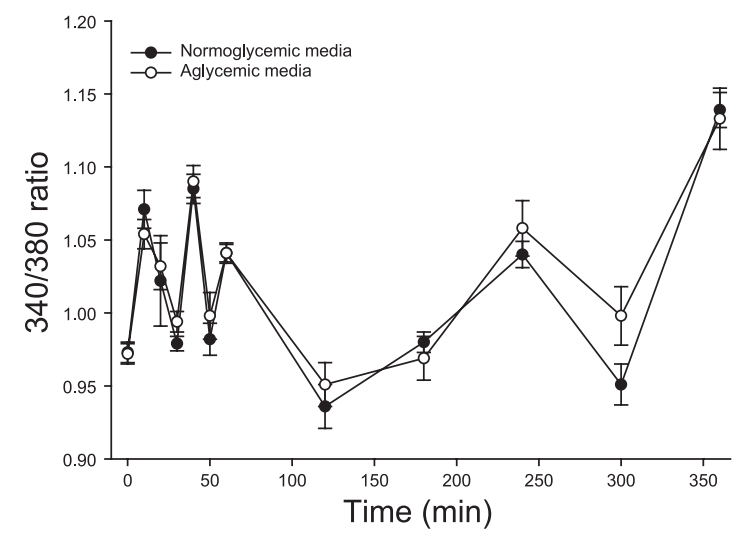

Fig. 2. Hypoxic stress causes a multiphasic increase in intracellular calcium in BBMEC. BBMEC were subjected to $6 \mathrm{~h}$ of hypoxic stress under normoglycemic or aglycemic conditions. Within the $1 \mathrm{st} \mathrm{h}$ of hypoxic exposure, there were 3 transient peaks in intracellular calcium. Calcium levels then returned to baseline and, subsequently, increased at 4 and $6 \mathrm{~h}$ of hypoxic stress. There was no statistical difference in intracellular calcium between normoglycemic and aglycemic conditions. A representative trace is shown. Values are means \pm SE; $n=8.340 / 380$ ratio, ratio of fluorescence at $340 \mathrm{~nm}$ to fluorescence at 380 $\mathrm{nm}$.
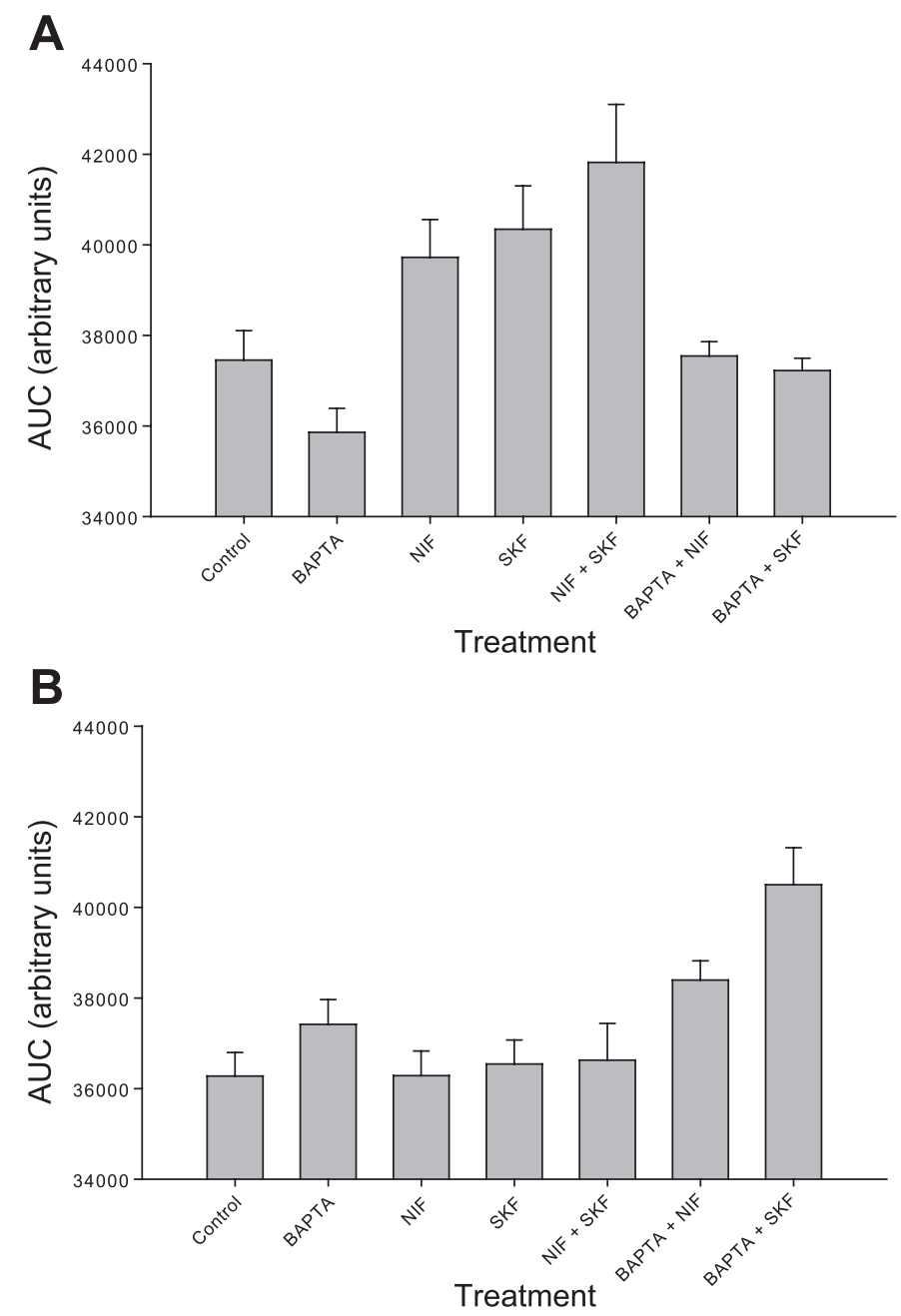

Fig. 3. Effect of BAPTA, nifedipine (NIF), and SKF-96365 (SKF) on intracellular calcium during $6 \mathrm{~h}$ of hypoxic stress. BBMEC were subjected to $6 \mathrm{~h}$ of hypoxia and incubated with $10 \mu \mathrm{M}$ BAPTA, $100 \mathrm{nM}$ NIF, or $100 \mathrm{nM}$ SKF, alone or in combination. There was a significant effect of drug treatment on intracellular calcium $\left(F_{6,604}=2.298, P=0.033\right)$ and a significant interaction between drug treatment and glycemic condition $\left(F_{6,604}=4.269, P<0.001\right)$. $A$ : under normoglycemic conditions, BAPTA decreased intracellular calcium. NIF and/or SKF increased the amount of intracellular calcium during the hypoxic stress. This effect of NIF and/or SKF was prevented by cotreatment with BAPTA. $B$ : under aglycemic conditions, there was no significant effect of BAPTA, NIF, or SKF, alone or in combination, on intracellular calcium levels, although BAPTA + NIF and BAPTA + SKF did potentially increase intracellular calcium. Values are means $\pm \mathrm{SE} ; n=6-20$. AUC, area under the curve.

To determine whether NIF or SKF could actually block changes in intracellular calcium, fura 2 fluorescence was measured after stimulation of BBMEC with bradykinin, a peptide known to trigger an increase in intracellular calcium in endothelial cells (17). After bradykinin stimulation under normoxic conditions, only SKF decreased the amount of intracellular calcium over the period of exposure to the peptide $\left(F_{3,30}=\right.$ $6.892, P=0.001$; Fig. 4), demonstrating that SKF-sensitive calcium influx pathways do exist in these cells.

BAPTA, NIF, and SKF partially protected against hypoxiainduced BBMEC monolayer disruption after hypoxic stress. BBMEC monolayers were exposed to hypoxic stress, and paracellular permeability was measured using $\left[{ }^{14} \mathrm{C}\right]$ sucrose. 


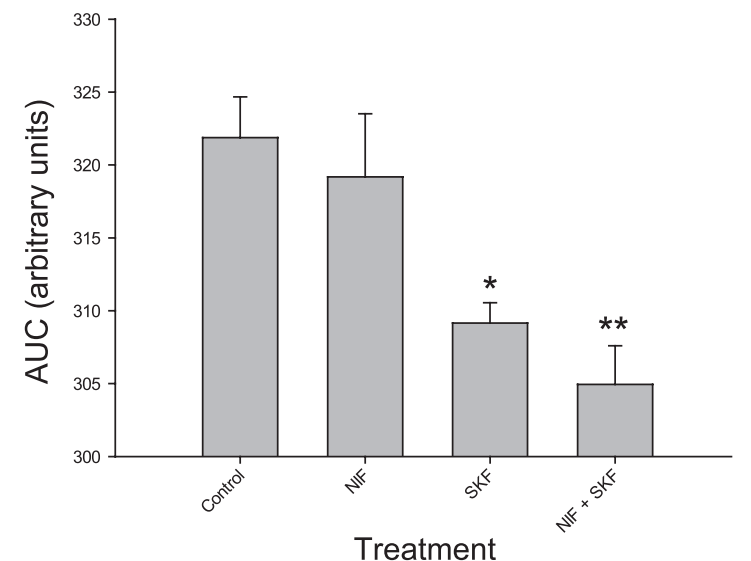

Fig. 4. SKF, but not NIF, prevents calcium influx after bradykinin stimulation. BBMEC were plated as described for hypoxic stress experiments. Cells were loaded with fura 2-AM and stimulated with $100 \mathrm{nM}$ bradykinin. Intracellular calcium was measured for $6 \mathrm{~h}$, and AUC was determined. One-way ANOVA indicated a significant blockade of bradykinin-stimulated calcium influx after SKF treatment $\left(F_{3,30}=6.892, P=0.001\right)$ but not after NIF treatment. Values are means \pm SE; $n=8-9$. $* P<0.05 ; * * P<0.01$ vs. control.

After $6 \mathrm{~h}$ of hypoxic stress, there was a significant effect of drug (BAPTA, NIF, or SKF) treatment $\left(F_{11,390}=4.232, P<\right.$ $0.001)$ and of hypoxic stress $\left(F_{1,390}=75.449, P<0.001\right)$ on BBB functional integrity. There was also a significant interaction between drug treatment and hypoxic stress $\left(F_{11,390}=\right.$ $1.851, P=0.044)$. Under normoxic-normoglycemic conditions, treatment with BAPTA, NIF, or SKF significantly decreased BBMEC monolayer permeability (Fig. 5A). Hypoxicnormoglycemic stress increased BBMEC monolayer permeability in almost all conditions. Statistical analyses indicate that only treatment with BAPTA + NIF protected against hypoxiainduced monolayer disruption.

Under normoxic-aglycemic conditions, there was no effect of any of the drug treatments on BBMEC monolayer permeability (Fig. 5B). Hypoxia caused an increase in monolayer permeability in control monolayers. Treatment with NIF or SKF alone prevented the hypoxia-induced permeability increase, but BAPTA treatment alone had no effect on this hyperpermeability. Monolayers treated with BAPTA + NIF were protected against hypoxia-induced increases in permeability, but BAPTA + SKF was not protective.

Antioxidant effects of NIF and SKF. Since NIF and SKF did not appear to act as calcium channel blockers in our hypoxia experimental paradigm (Fig. 3), we investigated whether the protective actions of these drugs were due to antioxidant properties. Cells were incubated with normoglycemic medium, aglycemic medium, or aglycemic medium with NIF or SKF as described for the calcium assays and permeability studies. ROS were measured using $2^{\prime}, 7^{\prime}$-dichlorofluorescein diacetate, a fluorescent probe that detects the presence of oxygen free radicals (12). BBMEC were subjected to $6 \mathrm{~h}$ of normoxia, $6 \mathrm{~h}$ of hypoxia, or $6 \mathrm{~h}$ of hypoxia followed by $30 \mathrm{~min}$ of reoxygenation in room air (Fig. 6). There was a significant effect of glucose and drug treatment $\left(F_{3,60}=19.213, P<0.001\right)$ and of oxygen treatment $\left(F_{2,60}=31.196, P<0.001\right)$ on ROS levels. Incubation in aglycemic medium alone increased ROS levels under any oxygen condition. ROS levels after $30 \mathrm{~min}$ of reoxygenation were significantly higher in all drug treatment groups than in the groups treated with $6 \mathrm{~h}$ of normoxia or $6 \mathrm{~h}$ of hypoxia. No antioxidant effect was observed with NIF or SKF under any of the three oxygen conditions.

\section{DISCUSSION}

Ischemic stroke and hypoxic stress disrupt the BBB (18). We previously demonstrated that hypoxic (49) or hypoxicaglycemic (1) stress can disrupt an in vitro BBB model system, providing an opportunity to investigate some of the cellular mechanisms underlying this disruption. Interestingly, two calcium channel blockers, NIF and SKF, can prevent BBB disruption in this model system (1). We chose to examine the role of intracellular calcium in modulating BBB functional perme-

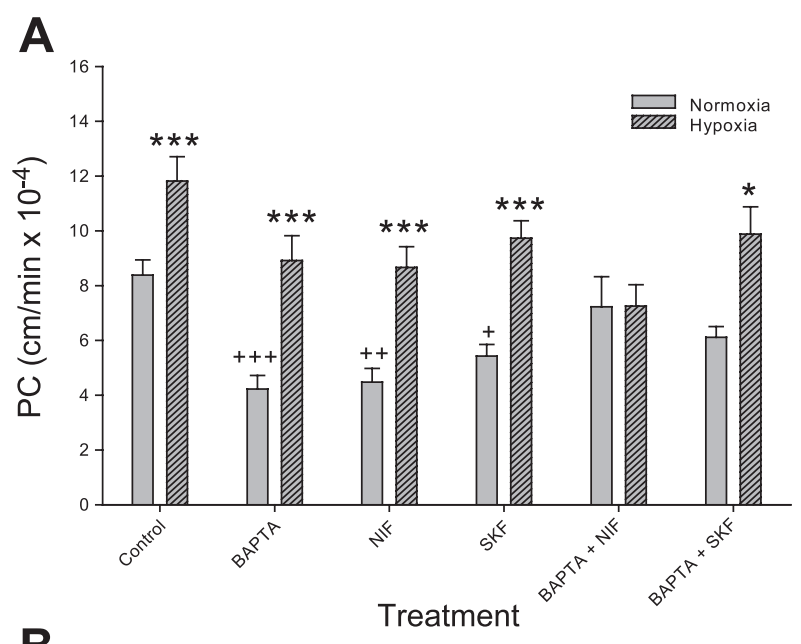

B

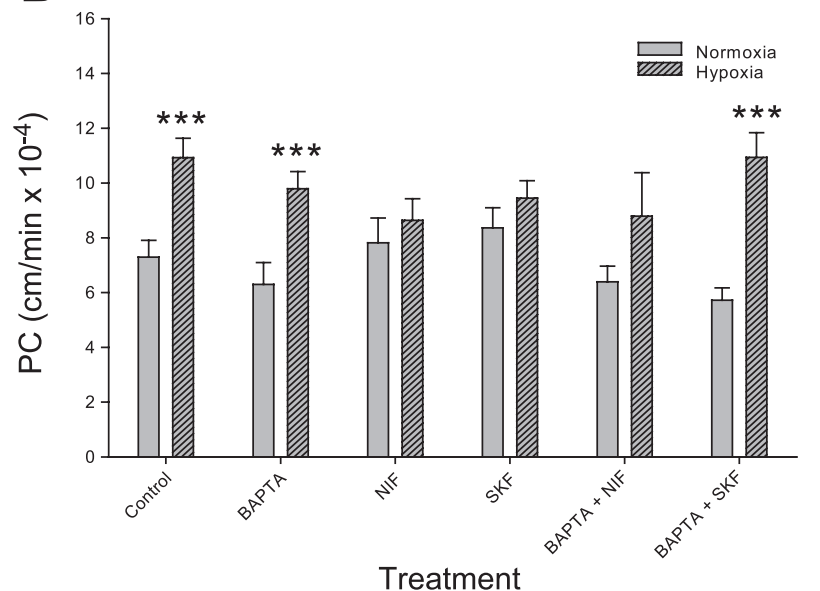

Fig. 5. BAPTA, NIF, and SKF partially protect BBMEC monolayer integrity. BBMEC monolayers were treated with $10 \mu \mathrm{M}$ BAPTA, $100 \mathrm{nM}$ NIF, and 100 $\mathrm{nM}$ SKF, alone or in combination. After $6 \mathrm{~h}$ of hypoxic stress, monolayer permeability was assayed. Two-way ANOVA indicated a significant effect of drug treatment $\left(F_{11,390}=4.232, P<0.001\right)$ and a significant effect of hypoxic stress $\left(F_{1,390}=75.449, P<0.001\right)$, as well as a significant interaction between drug treatment and hypoxic stress $\left(F_{11,390}=1.851, P=0.044\right)$. A: pairwise comparisons indicate that, under normoxic/normoglycemic conditions, BAPTA and NIF treatment significantly reduced monolayer permeability compared with normoxic control. Treatment with BAPTA, NIF, SKF, or BAPTA + SKF did not block the hypoxia-induced increase in monolayer permeability. Only BAPTA + NIF prevented monolayer disruption. $B$ : in BBMEC subjected to aglycemic conditions, NIF and SKF prevented hypoxiainduced increases in permeability, as did BAPTA + NIF. Neither BAPTA alone nor BAPTA + SKF prevented this hypoxic effect. Values are means \pm SE; $n=6-30 . * * * P<0.001$ vs. normoxia. ${ }^{+++} P<0.001 ;{ }^{++} P<0.01$; ${ }^{+} P<0.05 ; * P<0.05$ vs. normoxic control. 


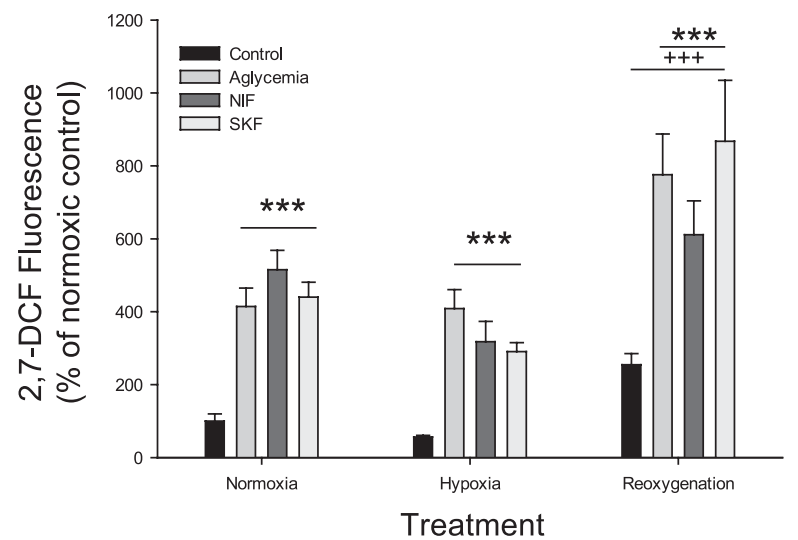

Fig. 6. NIF and SKF are not antioxidants in this system. BBMEC were plated and subjected to $6 \mathrm{~h}$ of hypoxic stress under normoglycemia, aglycemia, or aglycemia with NIF or SKF treatment. Production of reactive oxygen species (ROS) was measured using $2^{\prime}, 7^{\prime}$-dichlorofluorescein diacetate (2,7-DCF). There was a significant effect of glucose and drug treatment $\left(F_{3,60}=19.213\right.$ $P<0.001)$ and a significant effect of oxygen $\left(F_{2,60}=31.196, P<0.001\right)$. Aglycemic treatment alone increased ROS in BBMEC. A 30-min reoxygenation period after $6 \mathrm{~h}$ of hypoxic stress also caused a significant increase in ROS. Treatment with NIF or SKF did not prevent this reoxygenation-induced increase in ROS. Values are means $\pm \mathrm{SE} ; n=10-12$. $* * * P<0.001$ vs normoglycemia (control). ${ }^{+++} P<0.001$ vs. normoxia.

ability and its potential involvement in mediating hypoxiainduced damage.

Although there is a large body of literature dealing with the role of calcium in modulating cell-cell adhesion through adherens junctions, much less is known about the role of calcium in modulating TJ function (13). We used a calcium ionophore to increase levels of intracellular calcium in our BBB model system and found that simply increasing intracellular calcium alone did not lead to increased paracellular permeability, indicating that high intracellular calcium levels alone, such as those seen after a stroke $(43,60)$ or hypoxic event $(35,41,55)$, are not sufficient to alter TJ function. However, there is evidence that TJ components, such as ZO-1 $(61,69)$ and occludin (55), are sensitive to calcium levels. It remains to be seen how increased intracellular calcium alters $\mathrm{TJ}$ protein expression or function in this experimental paradigm.

We found that a 6-h hypoxic or hypoxic-aglycemic stress triggered a characteristic increase in intracellular calcium in BBB endothelial cells. Within the $1 \mathrm{st} h$ of exposure to the stressor, intracellular calcium levels displayed distinct oscillations with several peaks, perhaps representing the initial response of BBB endothelial cells to stress. This initial response was followed by increases in intracellular calcium at 4 and $6 \mathrm{~h}$, which may reflect long-term slow calcium oscillatory behavior. There was no difference in the calcium response between cells incubated in aglycemic medium and those incubated in normoglycemic medium during hypoxic stress. However, the presence or absence of glucose did influence cellular responses to the calcium chelator (BAPTA) or calcium channel blockers (NIF or SKF). Under normoglycemic conditions, treatment with NIF and/or SKF increased intracellular calcium levels over the course of the hypoxic stress. This result is in direct opposition to the presumed mechanism of action of these drugs: NIF is an L-type calcium channel blocker, while SKF blocks some stores-operated calcium channels. Under aglycemic conditions, intracellular calcium levels were not signifi- cantly altered, regardless of drug treatment. However, these cells do have some SKF-sensitive calcium channels, as indicated by the bradykinin experiment (Fig. 4), although these channels may not be involved in mediating hypoxic effects.

There is debate about the presence of L-type calcium channels on endothelial cells, a nonexcitable cell type. A few studies have demonstrated L-type channel currents in endothelial cells (64), and there is pharmacological evidence that these drugs prevent increases in intracellular calcium in endothelial cells (67) and protect against hypoxia-induced damage $(1,29)$; however, there is no convincing molecular evidence for the presence of these channels at the BBB. Therefore, we must consider the possibility that the protective effect of NIF seen in BBB models is mediated through an alternate mechanism. The present study and others have demonstrated that NIF has protective effects under hypoxic conditions. NIF can inhibit hypoxic damage by inhibiting protein kinase (PKC)- $\alpha$ $(\mathrm{PKC}-\alpha)(29)$, but this does not correlate with the increase in intracellular calcium (potentially activating PKC- $\alpha$ ) seen after treatment with NIF in this study and in others $(8,63)$.

SKF is considered to be a stores-operated calcium channel blocker, but its parent compound, SC-38249, can also inhibit extrusion of intracellular calcium via the plasma membrane $\mathrm{Ca}^{2+}$-ATPase (PMCA) (16). SKF does block an increase in endothelial cell calcium after stimulation with bradykinin (22), as we have demonstrated in this study. However, other studies have demonstrated that treatment with SKF alone can trigger an increase in intracellular calcium that is mediated by release from intracellular, thapsigargin-sensitive stores (36) and by influx from the extracellular medium (38), suggesting that SKF may have dose- and cell system-specific effects on intracellular calcium.

What is the potential source of the signal triggering this increase in intracellular calcium under hypoxic-normoglycemic conditions? Why is such an increase not observed under hypoxic-aglycemic conditions? One potential trigger for intracellular calcium increases under these hypoxic-normoglycemic conditions is nitric oxide (NO) produced by calcium-dependent stimulation of endothelial NO synthase (eNOS) and released from surrounding endothelial cells. NO is produced by cerebral microvessel endothelial cells under hypoxic stress (48), which increases $\mathrm{BBB}$ endothelial cell permeability $(10,48)$; this effect is attenuated by inhibition of NOS (48). NO can also trigger an increase in intracellular calcium in endothelial cells $(6,9)$. Calcium is a major regulator of eNOS, a major source for NO production in these cells (23). Under our experimental conditions, hypoxic stress could trigger an initial increase in intracellular calcium that could activate eNOS to produce NO. This NO could act on the endothelial cell in a paracrine or autocrine fashion, thereby further increasing the level of intracellular calcium. The NO-mediated calcium increase would not be affected by NIF or SKF but would be subject to chelation by BAPTA, as seen in these studies (Fig. 3A). Furthermore, NIF treatment can upregulate eNOS protein levels (19) and stimulate endothelial NO production $(19,20,24)$, further implicating NO in this experimental paradigm. SKF treatment has not been convincingly shown to stimulate NO production on its own (36), but it may inhibit the activity of the PMCA (16), thereby prolonging any increase in intracellular calcium. Under aglycemic conditions, energy-dependent mechanisms may be inhibited, leading to prevention of eNOS function, and, thus, may 
prevent the potentially NO-induced increase in intracellular calcium.

There are other mechanisms by which endothelial cells can increase intracellular calcium levels via pathways distinct from store-operated or voltage-gated channels. Hypoxia triggers expression of endothelin-1 (ET-1) (31) and vascular endothelial growth factor $(34,44)$. ET-1 can increase intracellular calcium (59) via an inositol trisphosphate-sensitive pathway (70); this increase can be potentiated by NOS inhibitors or decreased by NO donors (15), indicating cross talk between these two pathways. NO can induce expression of vascular endothelial growth factor, which in turn activates eNOS (11, 51), feeding into the NO pathway described above. The BBB endothelial cell also expresses a large number of ion transporters, the function of which may be altered under pathological conditions. One of these, the $\mathrm{Na}^{+} / \mathrm{Ca}^{2+}$ exchanger (NCX), can work in reverse (i.e., calcium influx coupled to sodium efflux) after pharmacological manipulation (21). Reversal of the NCX has been shown to be important in mediating spinal cord white matter injury after anoxia (46), and NCX inhibitors can protect against ischemic injury attributed to exchanger reversal (68). Furthermore, reversal of the NCX in endothelial cells has been linked to activation of eNOS and production of $\mathrm{NO}(57,62)$. If the NCX in BBB endothelial cells is reversed under hypoxic conditions, leading to calcium overload inside the cells, this might also explain why NIF and SKF do not block the increase in intracellular calcium after hypoxic stress. The data presented in this study may also indicate a secondary effect of drug treatment on the activity or expression of ion transporters critical for the removal of intracellular calcium, such as the NCX or PMCA (65), the expressions of which can be directly modulated by changes in intracellular calcium $(27,40)$.

As previously demonstrated, NIF protects against hypoxiainduced BBB functional disruption, and we confirmed the protective effect of SKF (1). Interestingly, treatment with BAPTA, NIF, or SKF under normoxia-normoglycemia actually tightened the monolayer, perhaps implicating resting intracellular calcium levels in TJ function. Under normoxicaglycemic conditions, there was no effect of drug treatment on monolayer permeability; however, NIF and SKF were able to completely prevent the hypoxia-induced increase in monolayer permeability under aglycemic conditions, while BAPTA had no protective effect. As with the A-23187 data, these results indicate that a dramatic alteration (an increase or a decrease) in intracellular calcium is not sufficient to disrupt or protect BBB monolayer functional integrity. Although other studies have proposed an antioxidant role for NIF $(7,47)$, we have ruled out the possibility that NIF or SKF is protective via an antioxidant mechanism in this system. As we have mentioned here, intracellular calcium levels are regulated by many cellular mechanisms, which makes it difficult to determine the exact mechanism by which calcium influences $\mathrm{TJ}$ and $\mathrm{BBB}$ monolayer permeability.

In conclusion, we examined the effects of BAPTA, NIF, and SKF treatment, alone or in combination, on BBB monolayer permeability, intracellular calcium levels, and ROS production after a hypoxic or hypoxic-aglycemic stress. We demonstrated an increase in intracellular calcium after NIF or SKF treatments under hypoxia-normoglycemia, in stark contrast to the presumed actions of these drugs to simply block calcium flux. The observed increase may reflect not a failure of the drugs to block their respective targets, which still occurs after bradykinin treatment, but, rather, an unmasking of calcium released from a secondary source, which may be crucial for mediating NIF and SKF protection of monolayer integrity. What remains to be determined in this experimental paradigm is the role of calcium in modulating $\mathrm{TJ}$ protein expression or protein-protein interactions.

We envision several mechanisms by which calcium could be involved in regulating BBB TJ function: 1 ) a direct interaction between calcium and TJ proteins (i.e., occludin) in a manner similar to that occurring with E-cadherin at the adherens junction (54), 2) a mechanism by which alterations in intracellular calcium levels, and consequent activation of signal transduction cascades (i.e., calcium-calmodulin kinases or PKC), directly regulate expression of TJ protein genes, and/or 3) an indirect mechanism by which calcium affects other proteins, such as eNOS, ET-1, ion transporters, or ATPases, thereby affecting BBB integrity. Future studies are targeted at determining the effect of changes in intracellular calcium on TJ protein expression, elucidating the source of the increased intracellular calcium after NIF or SKF treatment, and investigating the signal transduction cascades triggered by the treatments described in these studies to clarify the role of calcium in $\mathrm{TJ}$ protein regulation and BBB function.

\section{ACKNOWLEDGMENTS}

We thank Drs. Roger O'Neil and Anne Wolka for reviewing the manuscript.

Present address of R. C. Brown: Dept. of Integrative Biology and Pharmacology, University of Texas Health Science Center at Houston, 6431 Fannin St., Houston, TX 77030 .

\section{GRANTS}

This work was supported by National Institute of Neurological Disorders and Stroke Grant NS-39592. R. C. Brown was supported by National Institute of Neurological Disorders and Stroke Grant F32 NS-43052 and K. S. Mark by Grant F32 NS-11175.

\section{REFERENCES}

1. Abbruscato TJ and Davis TP. Combination of hypoxia/aglycemia compromises in vitro blood-brain barrier integrity. J Pharmacol Exp Ther 289: 668-675, 1999.

2. Abbruscato TJ, Thomas SA, Hruby VJ, and Davis TP. Blood-brain barrier permeability and bioavailability of a highly potent and $\mu$-selective opioid receptor antagonist, CTAP: comparison with morphine. J Pharmacol Exp Ther 280: 402-409, 1997.

3. Audus KL and Borchardt RT. Characterization of an in vitro bloodbrain barrier model system for studying drug transport and metabolism. Pharm Res 3: 81-87, 1986.

4. Banks WA, Jaspan JB, and Kastin AJ. Effect of diabetes mellitus on the permeability of the blood-brain barrier to insulin. Peptides 18: 1577-1584, 1997.

5. Belayev L, Busto R, Zhao W, and Ginsberg MD. Quantitative evaluation of blood-brain barrier permeability following middle cerebral artery occlusion in rats. Brain Res 739: 88-96, 1996.

6. Berkels R, Dachs C, Roesen R, and Klaus W. Simultaneous measurement of intracellular $\mathrm{Ca}^{2+}$ and nitric oxide: a new method. Cell Calcium 27: 281-286, 2000.

7. Berkels R, Egink G, Marsen TA, Bartels H, Roesen R, and Klaus W. Nifedipine increases endothelial nitric oxide bioavailability by antioxidative mechanisms. Hypertension 37: 240-245, 2001.

8. Berkels R, Mueller A, Roesen R, and Klaus W. Nifedipine and Bay K 8644 induce an increase of $\left[\mathrm{Ca}^{2+}\right]_{\mathrm{i}}$ and nitric oxide in endothelial cells. J Cardiovasc Pharmacol 4: 175-181, 1999.

9. Berkels R, Suerhoff S, Roesen R, and Klaus W. Nitric oxide causes a cGMP-independent intracellular calcium rise in porcine endothelial cells-a paradox? Microvasc Res 59: 38-44, 2000. 
10. Boje KM and Lakhman SS. Nitric oxide redox species exert differential permeability effects on the blood-brain barrier. J Pharmacol Exp Ther 293: 545-550, 2000.

11. Brouet A, Sonveaux P, Dessy C, Balligand JL, and Feron O. Hsp90 ensures the transition from the early $\mathrm{Ca}^{2+}$-dependent to the late phosphorylation-dependent activation of the endothelial nitric-oxide synthase in vascular endothelial growth factor-exposed endothelial cells. J Biol Chem 276: 32663-32669, 2001.

12. Brown RC, Cascio C, and Papadopoulos V. Pathways of neurosteroid biosynthesis in cell lines from human brain: regulation of dehydroepiandrosterone formation by oxidative stress and $\beta$-amyloid peptide. J Neurochem 74: 847-859, 2000.

13. Brown RC and Davis TP. Calcium modulation of adherens and tight junction function: a potential mechanism for blood-brain barrier disruption after stroke. Stroke 33: 1706-1711, 2002.

14. Brown RC, Mark KS, Egleton RD, Huber JD, Burroughs A, and Davis TP. Protection against hypoxia-induced increase in blood-brain barrier permeability: role of tight junction proteins and NFkB. J Cell Sci 116: 693-700, 2003.

15. Chen Y, McCarron RM, Azzam N, Bembry J, Reutzler C, Lenz FA, and Spatz M. Endothelin-1 and nitric oxide affect human cerebromicrovascular endothelial responses and signal transduction. Acta Neurochir Suppl (Wien) 76: 131-135, 2000.

16. Ciardo A and Meldolesi J. Multiple actions of SC 38249: the blocker of both voltage-operated and second messenger-operated $\mathrm{Ca}^{2+}$ channels also inhibits $\mathrm{Ca}^{2+}$ extrusion. Eur J Pharmacol 188: 417-421, 1990.

17. Crawford KM, MacCallum DK, and Ernst SA. Agonist-induced $\mathrm{Ca}^{2+}$ mobilization in cultured bovine and human corneal endothelial cells. Curr Eye Res 12: 303-311, 1993.

18. Del Zoppo GJ and Hallenbeck JM. Advances in the vascular pathophysiology of ischemic stroke. Thromb Res 98: 73-81, 2000.

19. Ding Y and Vaziri N. Nifedipine and diltiazem but not verapamil up-regulate endothelial nitric-oxide synthase expression. J Pharmacol Exp Ther 292: 606-609, 2000.

20. Djuric D, Mitrovic V, and Jakovljevic V. The effects of calcium channel antagonists on coronary nitrite outflow in isolated rat heart. Arzneimittelforschung 52: 365-370, 2002.

21. Domotor E, Abbott NJ, and Adam-Vizi V. $\mathrm{Na}^{+}-\mathrm{Ca}^{2+}$ exchange and its implications for calcium homeostasis in primary cultured rat brain microvascular endothelial cells. J Physiol 515: 147-155, 1999.

22. Easton A and Abbott N. Bradykinin increases permeability by calcium and 5-lipoxygenase in the ECV304/C6 cell culture model of the bloodbrain barrier. Brain Res 953: 157-169, 2002.

23. Fleming I and Busse R. Molecular mechanisms involved in the regulation of the endothelial nitric oxide synthase. Am J Physiol Regul Integr Comp Physiol 284: R1-R12, 2003.

24. Fukuo K, Yang J, Suzuki T, Kaimoto T, Takemura Y, Yasuda O, Suhara T, Sato N, and Ogihara T. Nifedipine upregulates manganese superoxide dismutase expression in vascular smooth muscle cells via endothelial cell-dependent pathways. Hypertens Res 26: 503-508, 2003.

25. Furuse M, Hirase T, Itoh M, Nagafuchi A, Yonemura S, and Tsukita S. Occludin: a novel integral membrane protein localizing at tight junctions. J Cell Biol 123: 1777-1788, 1993.

26. Furuse M, Sasaki H, Fujimoto K, and Tsukita S. A single gene product, claudin-1 or -2 , reconstitutes tight junction strands and recruits occludin in fibroblasts. J Cell Biol 143: 391-401, 1998.

27. Guerini D, Garcia-Martin E, Gerber A, Volbracht C, Leist M, Merino CG, and Carafoli E. The expression of plasma membrane $\mathrm{Ca}^{2+}$ pump isoforms in cerebellar granule neurons is modulated by $\mathrm{Ca}^{2+}$.J Biol Chem 274: 1667-1676, 1999.

28. Hawkins CP, Mackenzie F, Tofts P, du Boulay EP, and McDonald WI. Patterns of blood-brain barrier breakdown in inflammatory demyelination. Brain Res 114: 801-810, 1991.

29. Hempel A, Lindschau C, Maasch C, Mahn M, Bychkov R, Noll T, Luft FC, and Haller H. Calcium antagonists ameliorate ischemia-induced endothelial cell permeability by inhibiting protein kinase C. Circulation 99: 2523-2529, 1999.

30. Hom S, Egleton RD, Huber JD, and Davis TP. Effect of reduced flow on blood-brain barrier transport systems. Brain Res 890: 38-48, 2001.

31. Hu J, Discher DJ, Bishopric NH, and Webster KA. Hypoxia regulates expression of the endothelin-1 gene through a proximal hypoxia-inducible factor-1 binding site on the antisense strand. Biochem Biophys Res Commun 245: 894-899, 1998.
32. Huber JD, Egleton RD, and Davis TP. Molecular physiology and pathophysiology of tight junctions in the blood-brain barrier. Trends Neurosci 24: 719-725, 2001.

33. Huber JD, Witt KA, Hom S, Egleton RD, Mark KS, and Davis TP. Inflammatory pain alters blood-brain barrier permeability and tight junctional protein expression. Am J Physiol Heart Circ Physiol 280: H1241H1248, 2001.

34. Ikeda E, Achen MG, Breier G, and Risau W. Hypoxia-induced transcriptional activation and increased mRNA stability of vascular endothelial growth factor in C6 glioma cells. J Biol Chem 270: 19761-19766, 1995.

35. Ikeda K, Nagashima T, Wu S, Yamaguchi M, and Tamaki N. The role of calcium ion in anoxia/reoxygenation damage of cultured brain capillary endothelial cells. Acta Neurochir Suppl (Wien) 70: 4-7, 1997.

36. Iouzalen L, Lantoine F, Pernollet M, Millanvoye-Van Brussel E, Devynck M, and Davis-Dufilho M. SK\&F 96365 inhibits intracellular $\mathrm{Ca}^{2+}$ pumps and raises cytosolic $\mathrm{Ca}^{2+}$ concentration without production of nitric oxide and von Willebrand factor. Cell Calcium 20: 501-508, 1996.

37. Itoh M, Furuse M, Morita K, Kubota K, Saitou M, and Tsukita S. Direct binding of three tight junction-associated MAGUKs, ZO-1, ZO-2, and ZO-3, with the COOH termini of claudins. J Cell Biol 147: 1351$1363,1999$.

38. Jan CR, Ho CM, Wu SN, and Tseng CJ. Multiple effects of 1-[ $\beta-[3-$ (4-methoxyphenyl)propoxy]-4-methoxyphenethyl]-1H-imidazole hydrochloride (SKF 96365) on $\mathrm{Ca}^{2+}$ signaling in MDCK cells: depletion of thapsigargin-sensitive $\mathrm{Ca}^{2+}$ store followed by capacitative $\mathrm{Ca}^{2+}$ entry, activation of a direct $\mathrm{Ca}^{2+}$ entry, and inhibition of thapsigargin-induced capacitative $\mathrm{Ca}^{2+}$ entry. Naunyn Schmiedebergs Arch Pharmacol 359: 92-101, 1999.

39. Kalaria RN. The blood-brain barrier and cerebrovascular pathology in Alzheimer's disease. Ann NY Acad Sci 893: 113-125, 1999.

40. Kaye DM and Kelly RA. Expression and regulation of the sodiumcalcium exchanger in cardiac microvascular endothelial cells. Clin Exp Pharmacol Physiol 26: 651-655, 1999.

41. Kimura C, Oike $\mathbf{M}$, and Ito Y. Hypoxia-induced alterations in $\mathrm{Ca}^{2+}$ mobilization in brain microvascular endothelial cells. Am J Physiol Heart Circ Physiol 279: H2310-H2318, 2000.

42. Kneisel $\mathbf{U}$ and Wolburg H. Tight junctions of the blood-brain barrier. Cell Mol Neurobiol 20: 57-76, 2000.

43. Kristian T and Siesjo BK. Calcium in ischemic cell death. Stroke 29: 705-718, 1998.

44. Kuo NT, Benhayon D, Przybylski RJ, Martin RJ, and LaManna JC. Prolonged hypoxia increases vascular endothelial growth factor mRNA and protein in adult mouse brain. J Appl Physiol 86: 260-264, 1999.

45. Kuroiwa T, Shibutani M, and Okeda R. Blood-brain barrier disruption and exacerbation of ischemic brain edema after restoration of blood flow in experimental focal cerebral ischemia. Acta Neuropathol (Berl) 76: 62-70, 1988

46. Li S, Jiang Q, and Stys PK. Important role of reverse $\mathrm{Na}^{+}-\mathrm{Ca}^{2+}$ exchange in spinal cord white matter injury at physiological temperature. J Neurophysiol 84: 1116-1119, 2000.

47. Mak IT, Boehme P, and Weglicki WB. Antioxidant effects of calcium channel blockers against free radical injury in endothelial cells. Correlation of protection with preservation of glutathione levels. Circ Res 70: 1099-1103, 1992.

48. Mark KS, Burroughs A, Brown RC, Huber JD, and Davis TP. Nitric oxide mediates hypoxia-induced changes in paracellular permeability of cerebral microvasculature. Am J Physiol Heart Circ Physiol 286: H174H180, 2004.

49. Mark KS and Davis TP. Cerebral microvascular changes in permeability and tight junctions induced by hypoxia-reoxygenation. Am J Physiol Heart Circ Physiol 282: H1485-H1494, 2002.

50. Mark KS and Davis TP. Stroke: development, prevention and treatment with peptidase inhibitors. Peptides 21: 1965-1973, 2000.

51. Mayhan WG. VEGF increases permeability of the blood-brain barrier via a nitric oxide synthase/cGMP-dependent pathway. Am J Physiol Cell Physiol 276: C1148-C1153, 1999.

52. Menzies SA, Betz AL, and Hoff JT. Contributions of ions and albumin to the formation and resolution of ischemic brain edema. J Neurosurg 78: 257-266, 1993.

53. Mitic LL, Schneeberger EE, Fanning AS, and Anderson JM. Connexin-occludin chimeras containing the ZO-binding domain of occludin 
localize at MDCK tight junctions and NRK cell contacts. J Cell Biol 146: 683-693, 1999.

54. Morita K, Sasaki H, Furuse M, and Tsukita S. Endothelial claudin: claudin-5/TMVCF constitutes tight junction strands in endothelial cells. J Cell Biol 147: 185-194, 1999.

55. Park JH, Okayama N, Gute D, Krsmanovic A, Battarbee H, and Alexander JS. Hypoxia/aglycemia increases endothelial permeability: role of second messengers and cytoskeleton. Am J Physiol Cell Physiol 277: C1066-C1074, 1999.

56. Reese TS and Karnovsky MJ. Fine structural localization of a bloodbrain barrier to exogenous peroxidase. J Cell Biol 34: 207-217, 1967.

57. Schneider J, El Kabir D, Chereau C, Mercier J, Dall'ava-Santucci J, and Dinh-Xuan A. Involvement of $\mathrm{Na}^{+} / \mathrm{Ca}^{2+}$ exchanger in endothelial NO production and endothelium-dependent relaxation. Am J Physiol Heart Circ Physiol 283: H837-H844, 2002.

58. Shasby DM and Shasby SS. Effects of calcium on transendothelial albumin transfer and electrical resistance. J Appl Physiol 60: 71-79, 1986.

59. Stanimirovic DB, Nikodijevic B, Nikodijevic-Kedeva D, McCarron RM, and Spatz M. Signal transduction and $\mathrm{Ca}^{2+}$ uptake activated by endothelins in rat brain endothelial cells. Eur J Pharmacol 288: 1-8, 1994.

60. Stevens H, Krop-van Gastel W, and Korf J. ${ }^{60} \mathrm{Co}$ and ${ }^{45} \mathrm{Ca}$ autoradiography in cerebral ischemia in the rat. Neurosci Lett 258: 183-186, 1998.

61. Stuart RO, Sun A, Panichas M, Hebert SC, Brenner BM, and Nigam SK. Critical role for intracellular calcium in tight junction biogenesis. J Cell Physiol 159: 423-433, 1994.

62. Teubl M, Groschner K, Kohlwein SD, Mayer B, and Schmidt K. $\mathrm{Na}^{+} / \mathrm{Ca}^{2+}$ exchange facilitates $\mathrm{Ca}^{2+}$-dependent activation of endothelial nitric-oxide synthase. J Biol Chem 274: 29529-29535, 1999.
63. Valencia L, Bidet M, Martial S, Sanchez E, Melendez E, Tauc M, Poujeol C, Martin D, Namorado M, Reyes J, and Poujeol P. Nifedipine-activated $\mathrm{Ca}^{2+}$ permeability in newborn rat cortical collecting duct cells in culture. Am J Physiol Cell Physiol 280: C1193-C1203, 2001

64. Vinet $\mathbf{R}$ and Vargas FF. L- and T-type voltage-gated $\mathrm{Ca}^{2+}$ currents in adrenal medulla endothelial cells. Am J Physiol Heart Circ Physiol 276: H1313-H1322, 1999.

65. Wang X, Reznick S, Li P, Liang W, and van Breemen C. $\mathrm{Ca}^{2+}$ removal mechanisms in freshly isolated rabbit aortic endothelial cells. Cell Calcium 31: 265-277, 2002.

66. Weber SJ, Abbruscato TJ, Brownson EA, Lipkowski AW, Polt R, Misicka A, Haaseth RC, Bartosz H, Hruby VJ, and Davis TP. Assessment of an in vitro blood-brain barrier model using several $\left[\mathrm{Met}^{5}\right.$ ]enkephalin opioid analogs. J Pharmacol Exp Ther 266: 1649-1655, 1993.

67. Yakubu MA and Leffler CW. L-type voltage-dependent $\mathrm{Ca}^{2+}$ channels in cerebral microvascular endothelial cells and ET-1 biosynthesis. Am J Physiol Cell Physiol 283: C1687-C1695, 2002.

68. Yamashita J, Itoh M, Kuro T, Kobayashi Y, Ogata M, Takaoka M, and Matsumura Y. Pre- or post-ischemic treatment with a novel $\mathrm{Na}^{+} /$ $\mathrm{Ca}^{2+}$ exchange inhibitor, KB-R7943, shows renal protective effects in rats with ischemic acute renal failure. J Pharmacol Exp Ther 296: 412-419, 2001.

69. Ye J, Tsukamoto T, Sun A, and Nigam SK. A role for intracellular calcium in tight junction reassembly after ATP depletion-repletion. Am J Physiol Renal Physiol 277: F524-F532, 1999.

70. Yokokawa K, Kohno M, Murakawa K, Yasunari K, and Takeda T. Effect of endothelin-1 on cytosolic calcium ions in cultured human endothelial cells. J Hypertens 8: 843-849, 1990.

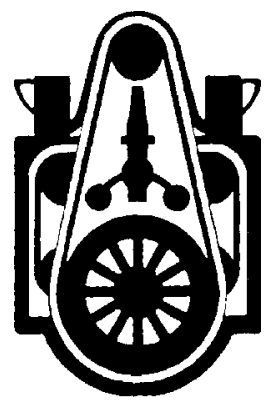

